\title{
ACCESO Y USABILIDAD DE MEDICAMENTOS: PROPUESTA PARA UNA DEFINICIÓN OPERACIONAL
}

\author{
Teodoro J. Oscanoa ${ }^{1, a}$
}

\begin{abstract}
RESUMEN
Las investigaciones sobre accesibilidad a medicamentos con encuestas a hogares, son de gran importancia para verificar la realidad y eficacia de las medidas de intervención para mejorar el acceso de medicamentos en la población. Desafortunadamente dichos estudios, además de ser escasos, presentan problemas metodológicos, que en su mayor parte son consecuencia de una ausencia de uniformidad en las definiciones operacionales de acceso y su diferenciación con las dimensiones de la accesibilidad. El objetivo de la propuesta es diferenciar ambos términos. Se propone definir operacionalmente acceso a medicamentos al proceso de verificación de la obtención de un fármaco por un paciente, independiente de diversos factores que pueden afectar el proceso. Se introduce el término "Usabilidad de fármacos", definiéndolo operacionalmente como los procesos destinados a medir las dimensiones de la accesibilidad de los medicamentos: disponibilidad física, asequibilidad, accesibilidad geográfica, aceptabilidad (o satisfacción).
\end{abstract}

Palabras clave: Comercialización de medicamentos; Utilización de medicamentos; Control de medicamentos y narcóticos (fuente: DeCS BIREME).

\section{ACCESS AND USABILITY TO MEDICATIONS: A PROPOSAL FOR AN OPERATIONAL DEFINITION}

\begin{abstract}
Research about accessibility to medicines through household surveys is very important in order to verify the reality and effectiveness of interventions done to increase the access of the population to the medications. Unfortunately, such studies, on top of being very few, have methodological problems, which mostly result from a lack of uniformity in the operational definitions of access and the differentiation with the dimensions of accessibility. The aim of this paper is to propose setting a difference between both terms. We propose an operational definition of access to medications as the process of verification of the purchase of a drug by a patient, independently from many factors that can affect this process. The term "usability of drugs" is introduced, defining it operationally as aimed at measuring the dimensions of the accessibility to the medications: physical availability, affordability, geographical accessibility, acceptability (or satisfaction).
\end{abstract}

Key words: Pharmaceutical trade; Drug utilization; Drug and narcotic control (source: MeSH NLM).

\section{INTRODUCCIÓN}

Los medicamentos constituyen una de las tecnologías sanitarias más importantes que han marcado la pauta del progreso humano, en relación con aspectos de prevención, tratamiento y rehabilitación de la salud. Como toda creación humana, este proceso tan sencillo puede llegar a ser uno de los más complicados y, por ello, requerir investigarlo, especialmente cuando el fármaco no llega en forma oportuna al paciente. Según la Organización Mundial de la Salud (OMS) cerca de un tercio de la población mundial no tiene acceso a medicamentos, especialmente en países subdesarrollados (1), por consiguiente, se requieren tomar medidas urgentes con el fin de combatir este problema.

Para conseguir este objetivo es necesario su estudio sistemático; para ello es importante las definiciones operacionales y el diseño de instrumentos e indicadores para medir el fenómeno y evaluar sus intervenciones. Uno de las formas más importantes de estudiar la accesibilidad a medicamentos es mediante las encuestas a hogares; a pesar de la trascendencia de estos estudios para comprender la realidad de la

\footnotetext{
1 Servicio de Geriatría, Hospital Nacional Guillermo Almenara Irigoyen, Essalud. Lima, Perú.

2 Facultad de Medicina Humana, Universidad Nacional Mayor de San Marcos. Lima, Perú.

a Médico internista, doctor en Medicina
} 
accesibilidad de los medicamentos y los resultados de las medidas de intervención, dichas investigaciones son escasas en número, presentan defectos en su metodología y carecen de definiciones operacionales uniformes, lo que hace difícil la comparación y el análisis de sus hallazgos (2). El objetivo del presente artículo, es revisar la definición operacional de accesibilidad a medicamentos utilizada en estudios de encuestas a hogares y proponer un enfoque alternativo con el fin de mejorar, precisar y sistematizar sus hallazgos.

\section{CONCEPTUALIZACIÓN Y DEFINICIÓN OPERACIONAL ACTUAL DE ACCESIBILIDAD A MEDICAMENTOS}

Existen dos abordajes conceptuales de acceso a medicamentos: la primera basada en el modelo de utilización de servicios; y la segunda, de la OMS, enfocado en los medicamentos esenciales. La definición operacional de acceso a medicamentos como su obtención, con o sin pago del usuario del fármaco prescrito ${ }^{(3)}$, necesariamente debe ser complementada incorporando las siguientes consideraciones: el acceso a medicamentos implica no solo su expedición sino también los servicios farmacéuticos, inclusión del fenómeno de automedicación, calidad del producto y del servicio per se, acceso a la información sobre el uso racional del medicamento, sostenibilidad y continuidad del servicio ${ }^{(4)}$. La OMS considera que un individuo tiene acceso a medicamentos esenciales cuando tales fármacos pueden ser obtenidos dentro de una distancia de viaje razonable (accesibilidad geográfica), estar disponibles en los centros de salud (disponibilidad física), costo razonable (financieramente posible) y su prescripción sea producto de un uso racional del medicamento.

La definición de la OMS está estructurada en cuatro de las cinco dimensiones del acceso a medicamentos descritas por Penchansky y Thomas (5): disponibilidad (incluye comodidad), accesibilidad, servicio, gasto y aceptabilidad. Por otro lado, medicamentos esenciales según la OMS son aquellos que satisfacen las necesidades prioritarias de salud en la población. La selección viene dada por: pertinencia para la salud pública, prueba de su eficacia y seguridad, y su eficacia comparativa en relación al costo ${ }^{(6)}$.

La OMS en sus objetivos destinados a propiciar un acceso equitativo a los medicamentos esenciales, ha propuesto un modelo de acción colectiva que debe fomentar el cumplimiento de cuatro puntos fundamentales ${ }^{(1)}$ :

a) Selección y uso racional de los medicamentos: con elaboración de listas de medicamentos fundamentadas a partir de guías de práctica clínica basadas en la mejor evidencia sobre efectividad, seguridad, calidad y costo-efectividad. Uso de una lista nacional de medicamentos esenciales para fines de adquisiciones, reembolsos, capacitación, donaciones y supervisión.

b) Precios asequibles: con el objetivo de garantizar que los gastos en medicamentos de los gobiernos, proveedores de salud y consumidores, sean sostenibles y a la vez tiendan a mantener la equidad e inclusión de poblaciones menos favorecidas económicamente. Entre las medidas apoyadas por la OMS están la implementación de políticas de medicamentos genéricos, producción local, información de precios, entre otras estrategias.

c) Financiamiento sostenible: a través de mecanismos de financiación equitativa, que van desde el apoyo gubernamental, el seguro social de salud y los métodos de transición (pago de bolsillo y copagos).

d) Sistemas fiables de suministro: que incorporen una combinación de servicios de suministro público y privado para asegurar la provisión regular de medicamentos esenciales.

\section{MEDICIÓN DEL ACCESO A MEDICAMENTOS}

La evaluación de los sistemas de atención sanitaria -según la perspectiva de Avedis Donabedian (7) -, puede ser enfocado y medido bajo tres formas: estructura, proceso y resultados. La accesibilidad a medicamentos del individuo o de la población, implica el funcionamiento de un sistema de gestión farmacéutico, que incluye los servicios farmacéuticos y los medicamentos en sí mismos; por consiguiente, puede considerarse legítima la aplicación de concepto Donabediano a los estudios de accesibilidad de medicamentos.

La estructura incluye las cualidades de los centros en los que la asistencia se produce, esto incluye las cualidades de los recursos materiales (facilidades, equipamiento y dinero), recursos humanos (número y calificación del personal) y la estructura organizativa (organización del equipo de salud, métodos de control de calidad y métodos de reembolso) ${ }^{(7)}$. Para los propósitos del estudio de accesibilidad a medicamentos, están incluidos la infraestructura o instalaciones, proveedores, organizaciones y sistemas que deben tener la capacidad adecuada para proporcionar los servicios que ofrecen. Debe anotarse que la evaluación del factor estructural del acceso a medicamentos, implica el estudio del grado en que las organizaciones e individuos cumplen con criterios estandarizados para participar en el mercado farmacéutico y del sistema de salud en general ${ }^{(4)}$. 
El proceso describe lo que en realidad se hace al dar y recibir la asistencia, es decir, es una medida que une los elementos estructurales con el resultado real. Por ejemplo, en la atención médica, incluye las actividades del paciente al buscar y llevar a cabo la asistencia y las actividades del facultativo al hacer el diagnóstico y recomendar o ejecutar un tratamiento. Para los propósitos del presente tópico, los estudios de procesos incluyen, los medicamentos prescritos, exámenes de laboratorio realizados, etc. Las evaluaciones de proceso se realizan bajo parámetros preestablecidos, por ejemplo, en la prescripción farmacológica son las guías de práctica clínicas basadas en la mejor evidencia sobre una enfermedad o condición determinada ${ }^{(4)}$.

La medición por resultados evalúa los efectos de la asistencia sanitaria en el estado de salud del paciente o de la población, el impacto puede ser determinado tanto por los puntos medios o resultados finales. Ejemplos de resultados finales medibles son las tasas de infección, morbilidad, mortalidad y satisfacción del paciente. De manera general, comparando las medidas de estructura, proceso y resultados, la afirmación según la cual los dos primeros son buenos predictores de un resultado positivo, y que de los tres, la medida de impacto o resultado es la más importante, deben tomarse con precaución. Es necesario el análisis ponderado y juicioso de cada uno de estas medidas, cuando se estudian la influencia de factores como la adherencia a la farmacoterapia y el curso natural de la enfermedad ${ }^{(4)}$.

\section{PROBLEMAS METODOLÓGICOS EN LOS ESTUDIOS DE ACCESO A MEDICAMENTOS MEDIANTE ENCUESTAS A HOGARES}

Los estudios de utilización y accesibilidad de medicamentos basados en encuestas a hogares, forman parte del nivel II de evaluación de resultados, de estructura y procesos (nivel I) de acuerdo con la Estrategia de Medicamentos Esenciales de la OMS (8). Este tipo de estudios tiene la particularidad de evaluar el acceso a medicamentos de forma muy específica, diferenciándola del acceso a los servicios de salud, es decir, estudia el acceso a medicamentos y sus barreras, en su estado puro. La importancia de la diferenciación entre el acceso a medicamentos del acceso a servicios de salud es ominosa, por ejemplo, cuando se evalúa la accesibilidad a los fármacos en pacientes con enfermedades crónicas (hipertensión arterial, diabetes, etc), donde la prescripción médica de fármacos es por tiempo prolongado, la accesibilidad a medicamentos es requerida con mayor urgencia que el acceso a los servicios de salud, suponiendo que el paciente se encuentre estable y compensado de su dolencia crónica ${ }^{(9)}$.
Los objetivos de este tipo de investigaciones son evaluar la existencia y el modo del acceso a los medicamentos, así como su utilización, costo y la carga en el presupuesto familiar ${ }^{(8)}$. A pesar de la aparente simplicidad de sus objetivos, la metodología de las investigaciones de accesibilidad de medicamentos con encuesta a hogares, está muy lejos de la estandarización. Paniz et al. publicaron en el año 2010, un estudio sistemático cuyo objetivo fue la revisión de la metodología utilizada en investigaciones sobre este tema ${ }^{(2)}$. Solo hallaron nueve investigaciones hasta el 2008, en las cuales hallaron los siguientes problemas relacionados a la definición operacional de acceso a medicamentos:

a. Falta de discriminación entre la definición operacional de acceso y el estudio de las dimensiones de la accesibilidad a medicamentos: la mayor parte de los estudios definieron el acceso a medicamentos como la obtención del mismo, con o sin pago del usuario del fármaco prescrito ${ }^{(3)}$. Debe recordarse que aparte de la definición de acceso a medicamento es muy importante diferenciarlo de estudios sobre las dimensiones de la accesibilidad, este último término se refiere a los diversos factores que afectan el acceso a medicamentos como son: disponibilidad física (physical availability), asequibilidad (affordability), accesibilidad geográfica y aceptabilidad (o satisfacción) ${ }^{(4)}$.

Un caso ilustrativo es cuando se empleó la siguiente pregunta para un estudio sobre acceso de medicamentos por enfermedades crónicas: “¿en los últimos 12 meses, ha tomado menos medicamentos de los prescritos por su médico debido a su costo?" (10). La pregunta puede ser legítima, pero mezcla las definiciones de acceso a medicamentos del estudio de las dimensiones de la accesibilidad; adicionalmente introduce la posibilidad de obtención de medicamentos pero en número insuficiente ( $¿$ acceso parcial a medicamentos?), el cual puede ser un fenómeno interesante de investigar pero según las bases de una farmacoterapéutica basada en la evidencia, los resultados positivos de una intervención farmacológica son predecibles solo cuando el acceso es completo, es decir, tiene el fármaco prescrito en número suficiente para cumplir la dosis y el tiempo indicado.

b. Falta de diferenciación de acceso a medicamentos con adherencia farmacológica: un estudio definió el acceso a medicamentos como adherencia farmacológica (11). La pregunta que indagaba adherencia fue: "en los últimos doce meses, Ud. ha tomado menos medicamentos de los recetados por su médico? A las personas que respondieron afirmativamente se les catalogó como no adherentes, a ellos se les repreguntó las razones y 
entre las alternativas de respuesta figuraban: costo, no disponibilidad de fármaco, no tener transporte o no gustar tomar medicamentos. Debe aclararse que la adherencia farmacológica tiene una definición operacional y varios instrumentos de evaluación y una de las razones de no adherencia es el no acceso a medicamentos que debe ser analizado en sus diferentes dimensiones.

La adherencia o cumplimiento terapéutico (adherencia, alianza terapéutica, observancia, adhesión, cooperación, concordancia o colaboración) se ha definido como la magnitud con la cual la conducta de un paciente, en relación con la toma de la medicación, el seguimiento de una dieta o la modificación de hábitos de vida, coincide con las instrucciones proporcionadas por el médico o personal sanitario (11). Existen varios instrumentos para medir adherencia farmacológica, muchos de ellos validados ${ }^{(12)}$, entre las más utilizadas en encuestas está el cuestionario de Morisky-Green ${ }^{(13)}$. Idealmente en el estudio aludido, debió analizarse la adherencia con un instrumento validado y luego ver el acceso y sus dimensiones en los calificados como no adherentes.

c. Uso de diversos instrumentos para estudios de Acceso de Medicamentos: Al revisar la pregunta que indagaba el acceso a medicamentos, de 9 estudios revisados, 8 tenían diferencias importantes en su expresión. Por consiguiente, actualmente puede afirmarse que no existe un instrumento validado para este tipo de investigaciones de tanta importancia en la salud pública. En el año 2008, Wirtz y col publicaron un interesante estudio sistemático que analiza las investigaciones llevadas a cabo en México sobre el acceso y uso de medicamentos, entre los años 1990 y 2004; sin duda, sus resultados son de gran importancia para tomar en cuenta en los cambios e intervenciones en la política de medicamentos no solo en México, sino en algunos países de Latinoamérica. Sin embargo, cuando se hace el análisis de las definiciones operacionales sobre acceso a medicamentos utilizadas, se hacen evidentes las mismas dificultades encontradas por Paniz et al. ${ }^{(2)}$, a pesar que estas últimas fueron realizadas en el contexto de investigaciones de accesibilidad con encuestas en hogares.

En primer lugar, una de las preguntas que debía contestar el estudio fue: "¿son los medicamentos accesibles en instituciones privadas y públicas y ellos están disponibles"?, sin duda el objetivo es concreto y plausible, pero mezcla el concepto de acceso con sus dimensiones (incluyendo solo una de ellas: disponibilidad, faltando el resto); también aclara que la definición de acceso que utiliza para esta revisión sistemática se refiere a costo, distribución y marketing. Segundo, entre las palabras claves de búsqueda no incluye "acceso a medicamentos", sencillamente porque este término no existe. Tercero, este estudio sistemático refleja fielmente el estado actual en lo referente a investigaciones sobre acceso a medicamentos, estos en su mayoría forman parte y de modo secundario de estudios de utilización de medicamentos, uso racional de medicamentos o adherencia; curiosamente de los 108 estudios analizados, solo 13 fueron sobre acceso a medicamentos y ninguno de ellos llevaba en su titulo la palabra "acceso o accesibilidad" (14).

Por todo lo anterior señalado, se puede concluir que la problemática de las investigaciones en acceso a medicamentos mediante encuestas a hogares, presenta serios problemas debido a la carencia de una metodología estándar, que parte desde una definición operacional clara hasta la falta de un instrumento validado para este fin.

\section{¿ES POSIBLE COMPARAR EL ACCESO A MEDICAMENTOS CON EL ACCESO AL MUNDO DEL INTERNET?}

El mundo de la web, caracterizada por su pragmatismo, enfoca este tópico definiendo operacionalmente acceso y usabilidad. La accesibilidad se define como la posibilidad de que un producto o servicio web pueda ser accedido y usado por el mayor número posible de personas, independientemente de las limitaciones propias del individuo o de las derivadas del contexto de uso ${ }^{(15,16)}$. Para mejorar la accesibilidad se siguen diversos protocolos como los recomendados por la Web Accessibility Initiative (WAI).

La usabilidad proviene del término en inglés usability y expresa la facilidad con que las personas pueden utilizar una herramienta particular o cualquier otro objeto fabricado por humanos con el fin de alcanzar un objetivo concreto. Otra definición de usabilidad lo describe como la medida en la cual un producto puede ser usado por usuarios específicos para conseguir objetivos específicos con efectividad, eficiencia y satisfacción en un contexto de uso concreto (17). Resumiendo, el acceso a internet es la medida del ingreso a la red y la usabilidad implica que una vez ingresado, uno se quede con una página web o programa muy amigable y que no le "haga pensar mucho" y que todo el esfuerzo sea dirigido al motivo de la consulta y no en el medio en sí. Por otro lado para conseguir una mejor usabilidad, se sigue las recomendaciones de su creador Jakob Nielsen ${ }^{(15,16)}$.

En el mundo informático se mide el grado de usabilidad de un sistema, bajo formas de medición empírica 
y relativa de la usabilidad del mismo. La medición empírica es mediante trabajo de campo; las mediciones tienen la característica de ser relativas porque no tienen el objetivo de calificar si un programa es bueno o malo, sino cuan cerca está de las metas planeadas o mediante la comparación con otros programas similares.

Debe aclarase que los términos de acceso y usabilidad en informática, todavía generan discusiones sobre las fronteras de cada uno de estos términos; la idea es que funcionan y se considera que su exacta distinción parece innecesaria. La accesibilidad debe ser entendida como 'parte de', y al mismo tiempo 'requisito para', la usabilidad.

\section{PROPUESTA DE DEFINICIONES OPERACIONALES DE ACCESO Y USABILIDAD DE MEDICAMENTOS}

Acceso a medicamentos (o accesibilidad a medicamentos) se debería definir operacionalmente como el proceso de verificación de la obtención de un fármaco por un paciente, independiente de diversos factores que pueden afectar el proceso. La condición del medicamento prescrito por un profesional de la salud, es importante porque si una persona toma medicamentos sin prescripción, es automedicación, un problema importante de estudiar, pero este debe tener su propio sistema y su diseño.

Por otro lado, los pacientes pueden estar enfermos y no tener acceso a la consulta médica ( $\mathrm{y}$ peor aun a medicamentos), este caso requiere un estudio de acceso al sistema de salud, un tema importante de investigar, pero de diseño también diferente. En los estudios sobre acceso de medicamentos el objetivo esencial debe ser la identificación de dos tipos de personas, los de acceso positivo y los de acceso negativo a los medicamentos. Operacionalmente, los primeros serían las personas que demuestran objetivamente o por afirmación la posesión de un medicamento, en cantidad suficiente para cumplir farmacoterapia instaurada. En caso de enfermedad crónica, por el tiempo determinado hasta renovación de una nueva receta, incluye la demostración de pertenencia a un programa que asegura la obtención del medicamento. El acceso negativo a medicamentos quedaría definida como aquellas personas que no demuestran objetivamente, o niegan, la posesión de un medicamento; incluye a los pacientes que muestran o refieren poseer una cantidad de fármaco insuficiente para cumplir farmacoterapia instaurada. En caso de enfermedad crónica, desconoce la forma de renovación de una nueva receta o niega o no demuestra la

Tabla 1. Indicadores y metodología de medición de acceso a medicamentos.

\begin{tabular}{|c|c|c|}
\hline $\begin{array}{c}\text { Dimensiones de la } \\
\text { accesibilidad }\end{array}$ & $\begin{array}{l}\text { Definición } \\
\text { operacional }\end{array}$ & Indicadores \\
\hline \multirow{5}{*}{ Disponibilidad física } & \multirow{5}{*}{$\begin{array}{l}\text { Relación entre el tipo y } \\
\text { la cantidad de fármaco } \\
\text { o servicio que se } \\
\text { necesita y el tipo y la } \\
\text { cantidad de producto o } \\
\text { servicio prestado. }\end{array}$} & Porcentaje de medicamentos vigentes (no vencidos) en stock. \\
\hline & & Porcentaje de fármacos fuera de stock. \\
\hline & & $\begin{array}{l}\text { Porcentaje de fármacos prescritos solicitados para su dispensación pero que no } \\
\text { fueron dispensados. }\end{array}$ \\
\hline & & $\begin{array}{l}\text { Porcentaje de establecimientos que disponen de una buena fuente de información } \\
\text { sobre fármacos, incluyendo guías de práctica clínica (GPC). }\end{array}$ \\
\hline & & Presencia de un servicio de información al consumidor. \\
\hline \multirow{4}{*}{ Asequibilidad } & \multirow{4}{*}{$\begin{array}{l}\text { Relación entre los } \\
\text { precios de los fármacos } \\
\text { o servicios y la } \\
\text { capacidad del usuario } \\
\text { para pagar por ellos. }\end{array}$} & $\begin{array}{l}\text { Promedio de la diferencia de precios entre un producto genérico y de marca para el } \\
\text { tratamiento de las condiciones del trazador. }\end{array}$ \\
\hline & & $\begin{array}{l}\text { Precios del sector público y los del sector privado, comparado con la media de los } \\
\text { precios internacionales de los medicamentos para las condiciones del trazador. }\end{array}$ \\
\hline & & $\begin{array}{l}\text { Número de días que debe trabajar el empleado público de más bajo salario para } \\
\text { pagar por un tratamiento completo para una condición o enfermedad trazadora. }\end{array}$ \\
\hline & & Porcentaje de la población cubierta por seguros o esquemas de prepago. \\
\hline \multirow{3}{*}{$\begin{array}{l}\text { Accesibilidad } \\
\text { geográfica }\end{array}$} & \multirow{3}{*}{$\begin{array}{l}\text { Relación entre la } \\
\text { ubicación del fármaco } \\
\text { (lugar de expedición) o } \\
\text { servicio y la ubicación } \\
\text { del usuario final del } \\
\text { producto o servicio. }\end{array}$} & $\begin{array}{l}\text { Porcentaje de hogares ubicados a más de cinco kilómetros (o posiblemente de viaje } \\
\text { más de una hora) de un centro de salud / farmacia que se espera que tenga siempre } \\
\text { los fármacos disponibles. }\end{array}$ \\
\hline & & $\begin{array}{l}\text { Promedio de minutos de tiempo total de espera durante la última visita a un centro } \\
\text { de salud. }\end{array}$ \\
\hline & & Promedio de horas de tiempo de funcionamiento de establecimientos de salud por día. \\
\hline \multirow[b]{2}{*}{$\begin{array}{l}\text { Aceptabilidad } \\
\text { (satisfacción) }\end{array}$} & \multirow{2}{*}{$\begin{array}{l}\text { Relación entre las } \\
\text { actitudes del usuario } \\
\text { y sus expectativas } \\
\text { sobre los productos } \\
\text { y servicios y las } \\
\text { características reales } \\
\text { de estos. }\end{array}$} & $\begin{array}{l}\text { Número de medicamentos de la lista de medicamentos esenciales que se encuentran } \\
\text { entre los diez fármacos más vendidos en el sector privado. }\end{array}$ \\
\hline & & $\begin{array}{l}\text { Satisfacción de los resultados con respecto a la última visita a la farmacia o lugar de } \\
\text { expedición de medicamentos. }\end{array}$ \\
\hline
\end{tabular}

Fuente: WHO (World Health Organization), MSH (Management Sciences For Health): Defining and Measuring Access to Essential Drugs, Vaccines, and Health Commodities. Report of the WHO-MSH Consultative Meeting.Ferney-Voltaire France WHO MSH; 2000. 
pertenencia a un programa que asegura la obtención del medicamento.

La usabilidad de los medicamentos debería significar lo que actualmente se conoce como las dimensiones de la accesibilidad. En concordancia con lo anterior, la usabilidad del medicamento quedaría definida como el estudio de los procesos destinados a medir las dimensiones de la accesibilidad de los medicamentos: disponibilidad física, asequibilidad, accesibilidad geográfica, aceptabilidad (o satisfacción) ${ }^{(4)}$ (Tabla 1).

Cada una de las dimensiones del acceso a medicamentos son atravesadas por una dimensión común: la calidad de los productos (fármacos) y servicios. Esta se mide a través del porcentaje de muestreo de medicamentos trazadores que no pasaron las pruebas de calidad, porcentaje de cumplimiento de los tratamientos de acuerdo con las guías de práctica clínica de enfermedades o condiciones trazadoras y, la existencia de una lista de medicamentos esenciales o formulario de medicamentos nacional (actualizado en los últimos cinco años), que reflejen las recomendaciones de las guías de práctica clínica en base a MBE (medicina basada en la evidencia)

Una de la características interesantes de la "importación" del término usabilidad a la esfera farmacológica, es que su similar de la web, lo emplea porque la usabilidad se mide (y la accesibilidad se verifica), es decir hay grados de usabilidad. Se utilizan pruebas de usabilidad, que en el campo farmacológico serían las investigaciones sobre los indicadores de las diferentes dimensiones de la accesibilidad, que son empíricas y relativas; es decir, son trabajos de campo y con una meta previamente planificada de cumplimiento del grado de accesibilidad. La métrica de la usabilidad en informática se mide bajo criterios de efectividad, eficiencia y satisfacción del usuario. De manera similar, las diferentes dimensiones de la accesibilidad y sus indicadores, corresponderían a la métrica de la usabilidad de los fármacos.

En síntesis, los estudios de accesibilidad a medicamentos, se deben dividir en dos: la primera parte implica la identificación si la persona tiene o no el medicamento prescrito (acceso a medicamentos) y la segunda, sobre todos los factores que influyen en los mismos (usabilidad de los medicamentos).

\section{LIMITACIONES DE LA PROPUESTA}

Existen varias limitaciones sobre la propuesta de modificar o aclarar las definiciones operacionales de acceso a medicamentos y la introducción del término usabilidad del fármaco. La primera, es que la palabra "usabilidad" no figura en el diccionario de la Real Academia Española de la Lengua ${ }^{(18)}$; sin embargo, debe anotarse que este término se emplea desde la década del 40 del siglo pasado, para describir la interacción humana con las máquinas. Otros términos relacionados no describen bien el concepto que se quiere transmitir: utilizable, manejable, servible, entre otros. Para los efectos comparativos que se quiere relacionar entre el mundo informático y farmacológico, solamente puede hacerse utilizando la palabra usabilidad.

Segundo, es posible argumentar que no es necesario introducir el término usabilidad del fármaco, debe quedarse como dimensiones de la accesibilidad, ya que según la propuesta la primera describe la segunda. La denominación de "dimensiones de la accesibilidad" como término (no como un constructo para el análisis del problema de acceso a medicamentos) es interesante y profundo, pero desafortunadamente es un verdadero fracaso, esto se hace evidente simplemente observando las investigaciones en este campo, cuyas dificultades ya fueron descritas. En el momento actual, solo existen dos alternativas, seguir y difundir el término para su mejor uso en las investigaciones, o cambiar el término a "usabilidad de los fármacos" y ver los resultados. ¿Dimensiones de la accesibilidad frente a usabilidad? Realmente necesitamos un término que no solo de la sensación de describir el fenómeno, sino que nos indique cual es el objetivo de la mejora del sistema que se analiza. En el mundo de la web este último término llama a la acción, si la usabilidad del software o página web es nula, este simplemente desaparece. En el mundo de los medicamentos, si los sistemas y procesos destinados a proveer de medicamentos no son "amigables" con el paciente, no está en juego la supervivencia de un software, sino la vida misma.

Tercero, puede considerarse inadecuado comparar el acceso al mundo de la web y los medicamentos, porque existe una diferencia casi insalvable: la decisión de ingresar (o acceder) es personal en el primero, sin embargo en el mundo del fármaco, existe un personaje intermedio y esencial, el profesional que prescribe el fármaco. Ambos mundos, tienen sus características, la propuesta no es una adopción sino una adaptación, creemos que la esencia básica se mantiene. Por otro lado, es importante aclarar que la definición operacional de acceso a medicamentos que se propone, solo considera a la población con prescripción médica exclusivamente. Bajo esta perspectiva el acceso al medicamento es un fenómeno posterior al acceso a un sistema de salud. Este argumento no invalida la propuesta, más bien la fortalece, en las investigaciones sobre acceso a medicamentos debe diferenciarse el 
acceso a medicamentos del acceso al sistema sanitario, dos sistemas muy interrelacionados y dependientes entre sí, pero para las investigaciones sobre su problemática es necesario diferenciarlas para mejorar las medidas de intervención.

El objetivo del acceso a medicamentos (con la definición que se propone) es tener cifras que analizar de cuántas personas no tienen disponible sus medicamentos a pesar que accedieron al médico prescriptor. El objetivo de estudio sobre la usabilidad de fármaco es para investigar todos los factores que intervienen en el proceso de obtención de un medicamento, con el fin de mejorar los procesos de un sistema farmacéutico de un país o lugar donde se realizó el estudio; este a su vez forma parte de un objetivo más grande que es la completa accesibilidad a los sistemas de salud de un país.

Cuarto, la palabra usabilidad de fármacos no está reconocida como palabra clave (key word) que facilita la búsqueda bibliográfica. En realidad el término "acceso a medicamentos" tampoco lo está, La mayoría de los estudios sobre este tópico se "esconden" bajo otras palabras claves existentes y aceptadas como estudio de utilización de medicamentos y acceso, pero este último referido a los servicios de salud y no a medicamentos ${ }^{(2)}$.

\section{CONCLUSIONES}

La accesibilidad a medicamentos es un problema grave para millones de personas en el mundo, especialmente en países en desarrollo y requiere de manera urgente investigaciones y de acuerdo con sus hallazgos, implementar las medidas de intervención adecuadas para solucionarlo. Los estudios sobre accesibilidad a medicamentos con encuestas a hogares, son importantes para verificar la realidad y eficacia de las medidas de intervención para mejorar el acceso de medicamentos en la población. Sin embargo, dichos estudios, además de ser escasos, presentan problemas metodológicos serios, uno de los principales es la no diferenciación entre la definición operacional entre el acceso a medicamentos y el estudio de las dimensiones de la accesibilidad.

El presente trabajo propone definir operacionalmente acceso a medicamentos al proceso de verificación de la obtención de un fármaco por un paciente, independiente de diversos factores que pueden afectar el proceso, dicho fármaco debe ser prescrito por un profesional de la salud autorizado. Se introduce el término "Usabilidad de fármacos", definiendo operacionalmente como los estudios o métodos destinados a medir las dimensiones de la accesibilidad de los medicamentos: disponibilidad física, asequibilidad, accesibilidad geográfica, aceptabilidad (o satisfacción). El objetivo de la propuesta es diferenciar ambos términos con el fin de mejorar y especificar la métrica de cada uno de ellos. El origen del enfoque, proviene de una adaptación de las definiciones operacionales sobre acceso y usabilidad de la web e internet.

\section{Fuentes de financiamiento}

Autofinanciado.

\section{Conflictos de interés}

El autor declara no tener conflictos de interés en la publicación de este artículo.

\section{REFERENCIAS BIBLIOGRÁFICAS}

1. World Health Organization. WHO Medicines Strategy: countries at the core 2004-2007. Geneva: WHO; 2004.

Tabla 2. Propuesta de definiciones operacionales para investigaciones sobre acceso a medicamentos basadas en encuestas a hogares.

\begin{tabular}{ll}
\hline Terminología & Definición operacional \\
\hline Acceso a medicamentos & $\begin{array}{l}\text { Proceso de verificación de la obtención de un fármaco por un paciente, independiente de diversos } \\
\text { factores que pueden afectar el proceso, dicho fármaco debe ser prescrito por un profesional de la } \\
\text { salud autorizado. }\end{array}$ \\
$\begin{array}{l}\text { Acceso positivo a } \\
\text { medicamentos }\end{array}$ & $\begin{array}{l}\text { Persona que demuestra objetivamente o por afirmación la posesión de un medicamento, en can- } \\
\text { tidad suficiente para cumplir la farmacoterapia prescrita. En caso de enfermedad crónica, por el } \\
\text { tiempo determinado hasta renovación de una nueva receta. Incluye la demostración de pertenencia } \\
\text { a un programa que asegura la obtención del medicamento. }\end{array}$ \\
$\begin{array}{l}\text { Acceso negativo a } \\
\text { medicamentos }\end{array}$ & $\begin{array}{l}\text { Persona que no demuestra objetivamente, o niega la posesión de un medicamento. Incluye a } \\
\text { los pacientes que muestran o refieren poseer una cantidad de fármaco insuficiente para cumplir } \\
\text { farmacoterapia instaurada. En caso de enfermedad crónica, desconoce la forma de renovación de } \\
\text { una nueva receta. Niega o no demuestra la pertenencia a un programa que asegura la obtención } \\
\text { del medicamento. }\end{array}$ \\
\hline Usabilidad de fármacos & $\begin{array}{l}\text { Estudios de procesos destinados a medir las dimensiones de la accesibilidad de los medicamen- } \\
\text { tos: disponibilidad física, asequibilidad, accesibilidad geográfica, aceptabilidad (o satisfacción). }\end{array}$ \\
\hline
\end{tabular}


2. Paniz VM, Fassa AG, Maia MF, Domingues MR, Bertoldi $A D$. Measuring access to medicines: a review of quantitative methods used in household surveys. BMC Health Serv Res. 2010;10:146.

3. Leyva-Flores R, Erviti-Erice J, Kageyama-Escobar $\mathrm{ML}$, Arredondo A. Prescripción, acceso y gasto en medicamentos entre usuarios de servicios de salud en México. Salud Publica Mex. 1998;40(1):24-31.

4. World Health Organization, Management Sciences for Health. Defining and Measuring Access to Essential Drugs, Vaccines, and Health Commodities. Report of the WHOMSH Consultative Meeting. Ferney-Voltaire, France: WHO$\mathrm{MSH} ; 2000$.

5. Penchansky R, Thomas JW. The concept of access: definition and relationship to consumer satisfaction. Med Care. 1981;19(2):127-40.

6. World Health Organization. Equitable Access to Essential Medicines: A Framework for Collective Action - WHO Policy Perspectives on Medicines, No. 8. Geneva: WHO; 2004.

7. Donabedian A. The quality of care. How can it be assessed? JAMA. 1988;260(12):1743-8.

8. World Health Organization. Using indicators to measure country pharmaceutical situations. Fact Book on WHO Level I and Level II monitoring indicators. Geneva: WHO; 2006.

9. Carvalho MFC. The polypharmacy in elderly in Sao Paulo -SABE Study- Health, Well-being and aging. [Tesis de Maestría] Sao Paulo, Brasil: Facultade de Saude Publica da Universidade de Sao Paulo; 2007.

10. Piette JD, Heisler M, Wagner TH. Cost-related medication underuse among chronically ill adults: the treatments people forgo, how often, and who is at risk. Am J Public Health. 2004;94(10):1782-7.
11. World Health Organization. Adherence to long-term therapies: evidence for action. Geneva: WHO; 2003.

12. Osterberg L, Blaschke T. Adherence to medication. $\mathrm{N}$ Engl J Med. 2005;353(5):487-97.

13. Morisky DE, Ang A, Krousel-Wood M, Ward HJ. Predictive validity of a medication adherence measure in an outpatient setting. J Clin Hypertens (Greenwich). 2008;10(5):348-54.

14. Wirtz VJ, Reich MR, Leyva Flores R, Dreser A. Medicines in Mexico, 1990-2004: systematic review of research on access and use. Salud Publica Mex. 2008;50 Suppl 4:S470-9.

15. Montero YH. Introducción a la Usabilidad. No Solo Usabilidad [Internet]. 2002 [citado 14 setiembre 2007]; (1): [aprox. 2 p.]. Disponible en: http://www.nosolousabilidad. com/articulos/introduccion usabilidad.htm

16. Montero YH, Martín Fernández FJ. Qué es la Accesibilidad web. No Solo Usabilidad [Internet]. 2003 [citado 14 setiembre 2007]; (2): [aprox. 2 p.]. Disponible en: http:// www.nosolousabilidad.com/articulos/accesibilidad.htm

17. Floría Cortés A. Pero... ¿qué es, realmente, la usabilidad? [Internet]. Madrid: Fundación Sidar; 2000 [citado 14 setiembre 2007]. Disponible en: http://www.sidar.org/recur/ desdi/traduc/es/visitable/quees/usab.htm

18. Real Academia Española. Diccionario de la Lengua Española [Internet]. 22a edición. Madrid: RAE; 2001 [citado 4 abril 2011]. Disponible en: http://buscon.rae.es/drael/

Correspondencia: Teodoro Julio Oscanoa Espinoza. Dirección: Av. Grau 800. Lima 13, Perú. Teléfono: (511) 3242983 anexo 4085.

Correo electrónico: teodoro.oscanoa@essalud.gob.pe

\section{Consulte la versión electrónica de la} Revista Peruana de Medicina Experimental y Salud Pública en www.pubmed.gov

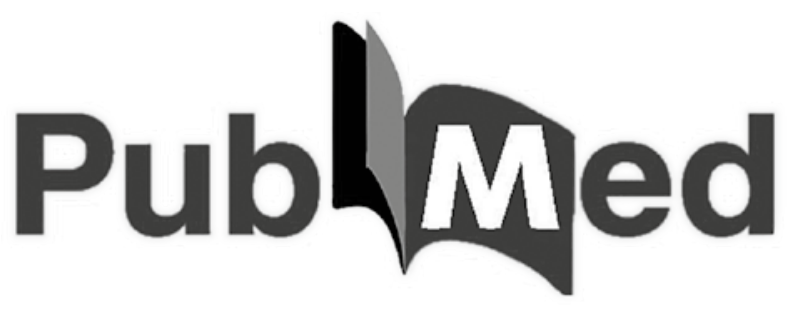

